\title{
Mediastinal vacuum phenomenon: atypical pneumomediastinum caused by gas replacement of diminished fat
}

This article was published in the following Dove Press journal:

International Medical Case Reports Journal

3 November 2015

Number of times this article has been viewed

\section{Hiroaki Hagiwara' \\ Ikuo Torii ${ }^{2}$}

'Department of Radiology, Yokohama Minamikyosai Hospital, ${ }^{2}$ Department of Radiology, Yokohama Municipal Citizen's Hospital, Yokohama, Japan
Correspondence: Hiroaki Hagiwara

Department of Radiology,

Yokohama Minamikyosai Hospital,

I-2I-I Mutsuura-higashi Kanazawa-ku,

Yokohama 236-0037, Japan

$\mathrm{Tel}+8 \mathrm{I} 457822101$

Fax +81457019159

Email hhagil936@gmail.com
Abstract: We report a case involving an 83-year-old man with interstitial lung disease who developed atypical pneumomediastinum caused by gas replacement of diminished fat. The patient presented with a complaint of worsening symptoms of respiratory difficulty since a diagnosis of chronic eosinophilic pneumonia 5 months back. He had been under observation with no particular treatment for 5 months. Computed tomography performed on admission revealed pneumomediastinum. When the current scan was compared with that obtained 5 months ago, it was evident that the fat surrounding the mediastinum had been replaced by gas density. There was no mediastinal enlargement, pneumothorax, or pneumopericardium. Because the patient was elderly, home oxygen therapy was initiated for the interstitial pneumonia with no steroid therapy. Computed tomography performed 10 months after discharge showed the reappearance of mediastinal fat and no evidence of gas density. This case is unique because the pneumomediastinum was distinct from spontaneous pneumomediastinum caused by alveolar air leaks and resembled the vacuum phenomenon caused by intervertebral disc degeneration.

Keywords: spontaneous pneumomediastinum, vacuum phenomenon, interstitial lung disease, anorexia nervosa

\section{Introduction}

Severe cough or hyperventilation can increase the thoracic pressure, leading to an abrupt increase in alveolar pressure and consequent rupture of alveoli. Subsequently, the air that leaks out may reach the mediastinum via the pulmonary hilum and bronchovascular interstitium. Therefore, pneumomediastinum is a pathology that involves the presence of free gas in the mediastinum due to different reasons. ${ }^{1}$ Spontaneous pneumomediastinum occurs in patients with interstitial lung disease and several other respiratory diseases. It generally appears in advanced stages of respiratory failure, and pneumothorax can occur if the air leakage due to increased thoracic pressure expands the mediastinum.

Here we report a case involving an 83-year-old man with interstitial lung disease who developed atypical pneumomediastinum caused by gas replacement of diminished fat due to generalized weight loss, with no mediastinal enlargement.

The pneumomediastinum observed in this case could not be explained by air leakage within the thorax, resembling the vacuum phenomenon caused by intervertebral disc degeneration, wherein gas accumulates in spaces created by decreased tissue volume in intervertebral discs and synovial joints. ${ }^{1}$

\section{Case report}

The patient reported here died 2 years ago, and it was difficult to contact the bereaved relatives; therefore, documented informed consent was waived by the Ethics Committee 
of Yokohama Minamikyosai Hopital. All procedures followed were in accordance with the ethical standards of the responsible committee on human experimentation (institutional and national) and with the tenets of the 1964 Helsinki Declaration and subsequent amended versions.

An 83-year-old man presented with worsening symptoms of respiratory difficulty since he was diagnosed with chronic eosinophilic pneumonia by another physician 5 months ago. At that time, computed tomography (CT) showed ground glass opacities and peripheral reticular opacities (Figure 1), while bronchoscopy revealed a $20 \%$ increase in eosinophils in his bronchoalveolar fluid. He was placed under observation with no particular treatment for 5 months, during which his symptoms only worsened; he eventually visited our institute for further examination.

On examination, the patient exhibited nonproductive cough, with no chest pain or painful swallowing. Chest auscultation revealed crackles at both lung bases, and blood tests for inflammatory markers showed normal findings.

Chest CT performed at the current examination showed a rapid decrease in mediastinal fat when compared with the scan obtained 5 months back, with the space created by the diminished fat replaced by gas density (Figure 2). The amount of subcutaneous fat also showed a decrease, indicating that the mediastinal fat loss was a part of generalized weight loss. Reticular structures considered to be fat remnants were observed with the regions of gas density, and there was no mediastinal enlargement, pneumothorax, or pneumopericardium. Widespread ground glass opacities and reticular opacities suggestive of interstitial pneumonia were observed below the pleura of both lungs.

The patient's symptoms continued to worsen. No specific treatment was provided for the pneumomediastinum, and,

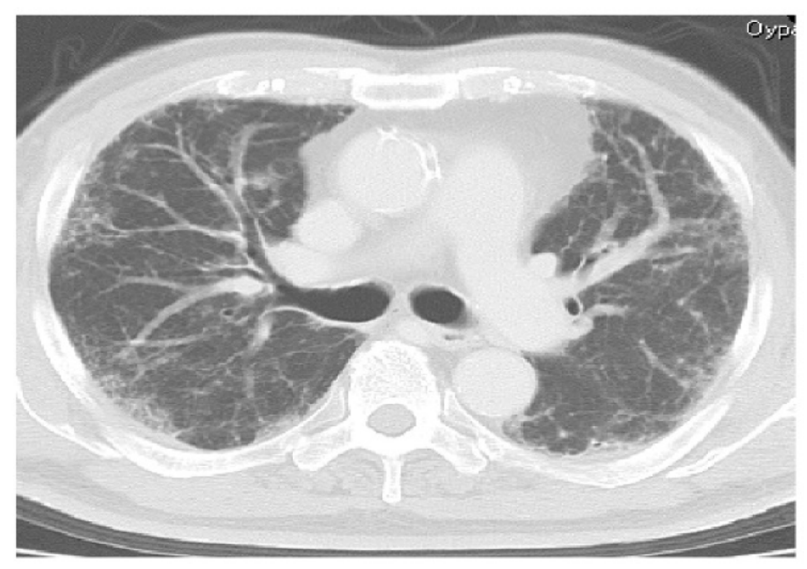

Figure I Chest computed tomography scan obtained 5 months before presentation. Note: Reticular shadows can be observed in the periphery of both lungs.

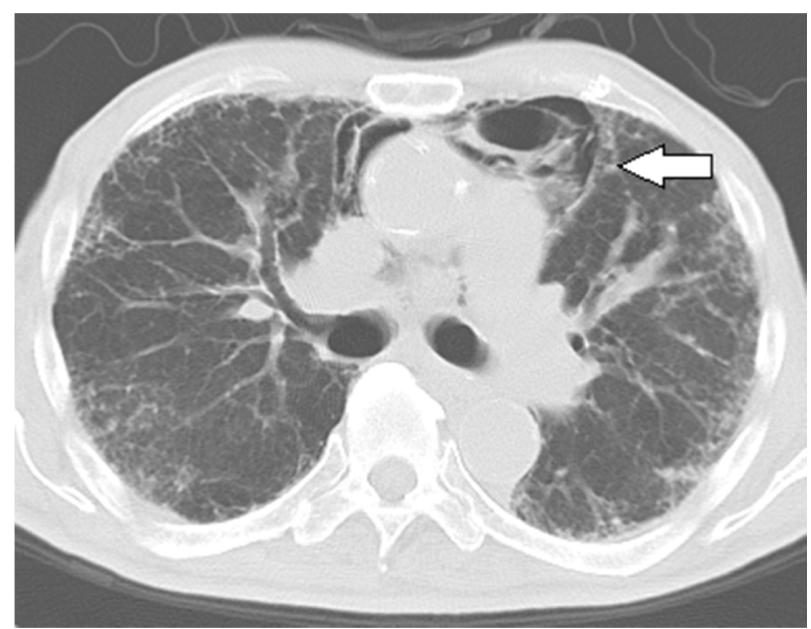

Figure 2 Chest computed tomography (CT) scan obtained on presentation to our institute.

Notes: Subcutaneous fat has decreased because of weight loss. The mediastinal fat observed on CT performed 5 months ago has been replaced by gas density (white arrow).

considering the patient's age, home oxygen therapy without steroid administration was initiated for the interstitial pneumonia. A routine follow-up CT performed 10 months after discharge showed some increase in subcutaneous and mediastinal fat and disappearance of the gas density in the mediastinum (Figure 3).

\section{Discussion}

Pneumomediastinum is etiologically categorized into spontaneous and secondary types. ${ }^{2}$ The pathophysiology of spontaneous pneumomediastinum involves a three-step process: alveolar rupture, air dissection along the bronchovascular sheaths, and spread of pulmonary interstitial emphysema into the mediastinum. ${ }^{3}$ Secondary pneumomediastinum, on

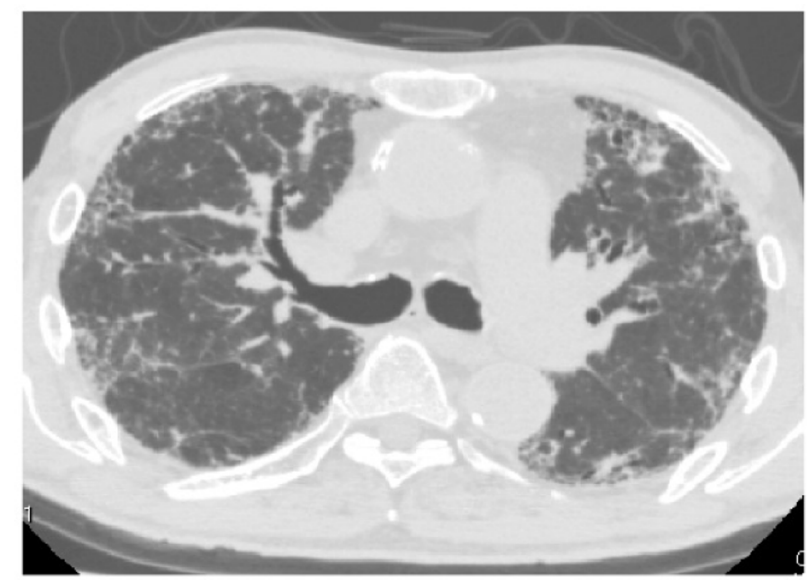

Figure 3 Chest computed tomography scan obtained at the 10-month follow-up visit. Note: An increase in subcutaneous and mediastinal fat is accompanied by disappearance of the gas density in the mediastinum. 
the other hand, can be iatrogenic or involve damage to the esophagus resulting from trauma, tracheobronchial damage, or infection by gas-producing bacteria. While secondary pneumomediastinum can be life threatening, the spontaneous type is associated with a good prognosis. ${ }^{4}$

Spontaneous pneumomediastinum has been reported to occur in patients with idiopathic interstitial fibrosis, other interstitial lung diseases, and connective tissue disorders. ${ }^{5,6}$ Increased thoracic pressure caused by cough or labored breathing may cause the alveoli to rupture. One report suggested that the use of corticosteroids for the treatment of underlying diseases weakens the interstitial lung tissues, ${ }^{7}$ while another suggested that spontaneous pneumomediastinum occurs if steroid or immunosuppressant therapy is not administered to patients on worsening of their condition. ${ }^{8}$ All these reports focused on air leaks as the underlying pathology.

However, we hypothesize that factors other than air leaks can cause pneumomediastinum on the basis of the findings from the present case. First, pneumomediastinum caused by air leaks is accompanied by mediastinal enlargement, which was not observed when CT scans obtained at a 5-month interval were compared. Furthermore, pneumatosis around the hilum of the lungs, which suggests the presence of air leaks, was not observed. Finally, the fat observed on previous CT scans was completely replaced by gas, and the shrunken space after fat loss was restored to the original form by gas accumulation, which is not observed in case of air leaks.

The vacuum phenomenon, which is characterized by the accumulation of gas in synovial joints or intervertebral discs, is a known pathology. Although the vacuum phenomenon is most frequently associated with degenerative joint disease, it is also associated with other pathologies; however, there is no consensus on its etiology. ${ }^{9}$ Bone fracture, necrosis, metastasis, abscess, and osteomyelitis are some of the described causes. The gas that accumulates in intervertebral discs contains at least $90 \%$ nitrogen, along with oxygen, carbon dioxide, and trace amounts of other gases. Unlike oxygen, nitrogen that elutes from dead spaces is not metabolized inside the body; rather, it accumulates in tissues as gas. ${ }^{10}$

We believe that the mediastinal gas collection in the present case was caused by the same pathology that causes the vacuum phenomenon. In healthy individuals, the lung volume increases when mediastinal fat decreases, and this leads to remodeling of the mediastinum and prevents gas accumulation. ${ }^{11}$

However, in interstitial pneumonia, restrictive disorders reduce the plasticity of the lungs, which inhibits remodeling in response to increased lung volume. We surmise that nitrogen elutes into the mediastinum when it is subjected to negative pressure during inspiration.

Spontaneous pneumomediastinum is mentioned as a complication of anorexia nervosa and other nonspecified eating disorders in several case reports. ${ }^{12}$ In all these reports, it was diagnosed when mediastinal gas accumulation was incidentally observed on radiographs or CT scans obtained at an arbitrary time point. None of the patients underwent surgical therapy, and all showed an improvement with conservative treatment. In an animal experiment, calorie restriction caused a decrease in the number of alveoli and thinning of the alveolar walls. ${ }^{13}$ These consequences increase the risk of alveolar rupture and spontaneous pneumomediastinum in malnourished patients with anorexia.

However, patients with eating disorders and our patient share a common factor associated with pneumomediastinum: weight loss. Among previous cases on spontaneous pneumomediastinum associated with eating disorders, some may have actually involved rapid disappearance of mediastinal fat followed by gas replacement, such as that observed in our case.

In conclusion, we reported a case of spontaneous pneumomediastinum resembling the vacuum phenomenon in an elderly patient with interstitial lung disease. The findings from this case suggest that spontaneous pneumomediastinum is not always associated with air leaks and mediastinal enlargement. Some cases, such as the present one, may be associated with a decrease in mediastinal fat in a given space, which is restored by gas accumulation. This pathology resembles the accumulation of nitrogen in intervertebral discs and vertebral bodies, that is, the vacuum phenomenon, in degenerative joint disease. Therefore, further examination or treatment is not necessary. Because this entity is different from spontaneous pneumomediastinum, we propose the term mediastinal vacuum phenomenon and consider it to be a form of secondary pneumomediastinum. Gas density in the mediastinum accompanying weight loss is observed not only in interstitial lung disease but also in anorexia; therefore, the possibility of a mediastinal vacuum phenomenon should be considered.

\section{Disclosure}

The authors report no conflicts of interest in this work.

\section{References}

1. Gohil I, Vilensky JA, Weber EC. Vacuum phenomenon: clinical relevance. Clin Anat. 2014;27(3):455-462.

2. Sahni S, Verma S, Grullon J, Esquire A, Patel P, Talwar A. Spontaneous pneumomediastinum: time for consensus. NAm J Med Sci. 2013;5(8): 460-464. 
3. Macklin MT, Macklin CC. Malignant interstitial emphysema of the lungs and mediastinum as an important occult complication in many respiratory diseases and other conditions: an interpretation of the clinical literature in the light of laboratory experiment. Medicine. 1944;23(4):281-358.

4. Bakhos CT, Pupovac SS, Ata A, Fantauzzi JP, Fabian T. Spontaneous pneumomediastinum: an extensive workup is not required. $\mathrm{J} \mathrm{Am} \mathrm{Coll}$ Surg. 2014;219(4):713-717.

5. Fujiwara T. Pneumomediastinum in pulmonary fibrosis. Detection by computed tomography. Chest. 1993;104(1):44-46.

6. Franquet T, Gimenez A, Torrubia S, Sabate JM, Rodriguez-Arias JM. Spontaneous pneumothorax and pneumomediastinum in IPF. Eur Radiol. 2000;10(1):108-113.

7. Yamanishi Y, Maeda H, Konishi F, et al. Dermatomyositis associated with rapidly progressive fatal interstitial pneumonitis and pneumomediastinum. Scand J Rheumatol. 1999;28(1):58-61.

8. Matsuoka S, Kurihara Y, Yagihashi K, Okamoto K, Niimi H, Nakajima Y. Thin-section CT assessment of spontaneous pneumomediastinum in interstitial lung disease: correlation with serial changes in lung parenchymal abnormalities. Respir Med. 2006;100(1):11-19.
9. Resnick D, Niwayama G, Guerra J Jr, Vint V, Usselman J. Spinal vacuum phenomena: anatomical study and review. Radiology. 1981;139(2):341-348.

10. Ford LT, Gilula LA, Murphy WA, Gado M. Analysis of gas in vacuum lumbar disc. AJR Am J Roentgenol. 1977;128(6):1056-1057.

11. Sengul AT, Sahin B, Celenk C, Basoglu A. Postoperative lung volume change depending on the resected lobe. Thorac Cardiovasc Surg. 2013;61(2):131-137.

12. Hunt DP, Becker AE, Guimaraes AR, Stemmer-Rachamimov A, Misdraji J. Case records of the Massachusetts General Hospital. Case 21-2012. A 27-year-old man with fatigue, weakness, weight loss, and decreased libido. N Engl J Med. 2012;367(2):157-169.

13. Massaro D, Massaro GD, Baras A, Hoffman EP, Clerch LB. Calorie-related rapid onset of alveolar loss, regeneration, and changes in mouse lung gene expression. Am J Physiol Lung Cell Mol Physiol. 2004;286(5):L896-L906.
International Medical Case Reports Journal

\section{Publish your work in this journal}

The International Medical Case Reports Journal is an international, peer-reviewed open-access journal publishing original case reports from all medical specialties. Previously unpublished medical posters are also accepted relating to any area of clinical or preclinical science. Submissions should not normally exceed 2,000 words or

\section{Dovepress}

4 published pages including figures, diagrams and references. The manuscript management system is completely online and includes a very quick and fair peer-review system, which is all easy to use. Visit http://www.dovepress.com/testimonials.php to read real quotes from published authors.

Submit your manuscript here: http://www.dovepress.com/international-medical-case-reports-journal-journal 